\title{
Atualização de Firmware em Sistemas Embarcados de Forma Segura e Confiável
}

\author{
Paulo Fylippe Sell ${ }^{1}$, Emerson Ribeiro de Mello ${ }^{1}$, Roberto de Matos ${ }^{1}$ \\ ${ }^{1}$ Instituto Federal de Santa Catarina (IFSC) \\ São José - SC - Brasil \\ paulo.fsealuno.ifsc.edu.br, \{mello, roberto.matos\}eifsc.edu.br
}

\begin{abstract}
Resumo. Uma atualização confiável de firmware garante que um dispositivo não ficará inutilizável ao final do processo. Uma atualização segura garante que somente firmware autênticos possam ser instalados no dispositivo. Fabricantes de microcontroladores possuem soluções próprias para uma atualização segura e confiável, porém cada solução possui particulares para uso. Este trabalho apresenta uma solução genérica para atualização segura e confiável de firmware de microcontroladores. Foi realizada uma implementação referência com o microcontrolador STM32L562QE de forma a validar a solução proposta e para demonstrar que é possivel obter atualização segura e confiável sem depender de soluções proprietárias dos fabricantes de microcontroladores.
\end{abstract}

\begin{abstract}
A reliable firmware update ensures that a device will not become unusable at the end of the process. A secure update ensures that only authentic firmware can be installed on the device. Microcontroller manufacturers have their own solutions for a secure and reliable update, however, each solution is tailored to its devices. This work presents a generic solution for a secure and reliable firmware update process. A reference implementation was performed using the STM32L562QE microcontroller in order to validate the proposed solution and to demonstrate that it is possible to obtain a safe and reliable upgrade without relying on proprietary solutions from microcontroller manufacturers.
\end{abstract}

\section{Introdução}

Mecanismos que permitem a atualização de firmware de forma remota poupam tempo e dinheiro, tanto do fabricante do dispositivo quanto do usuário, uma vez que o dispositivo não precisaria ser encaminhado ao fabricante para realizar o procedimento de atualização sempre que uma nova versão for lançada, seja para corrigir problemas ou para incluir novas funcionalidades ao mesmo [Jain et al. 2016]. Dispositivos conectados à Internet podem possuir soluções automatizadas que permitem realizar a atualização de forma espontânea, ou seja, sem qualquer intervenção do usuário. Porém, para os dispositivos que não possuem tal facilidade, o usuário é o responsável por baixar manualmente o arquivo do novo firmware, disponibilizado por exemplo no site web do fabricante, e realizar manualmente o processo de atualização no dispositivo.

A fim de que seja possível atualizar o firmware do dispositivo, uma pequena aplicação, chamada bootloader, deve ser embarcada no equipamento. O bootloader é invocado sempre que o dispositivo é energizado e seu principal papel é carregar o firmware 
instalado, sendo este último a aplicação que contém toda a lógica com as funcionalidades que aquele dispositivo deverá prover. O bootloader também pode ser responsável pelo processo de atualização de firmware e deve garantir que a atualização seja confiável e, em alguns cenários, segura. Para tanto, o bootloader deve ser imutável, além de ser capaz de se comunicar com outros dispositivos para que seja possível receber novos firmware [Beningo 2015].

Um processo de atualização de firmware é considerado confiável se, ao término do processo, o dispositivo conseguir reiniciar corretamente com o novo firmware, em caso de sucesso na atualização, ou executar o firmware previamente instalado, em caso de insucesso [Nikolov 2018]. O bootloader é o responsável por receber o novo firmware, verificar se o mesmo está íntegro, usando por exemplo funções hash criptográficas, e por fim, atualizar os ponteiros internos para que o novo firmware seja usado nas próximas inicializações do dispositivo.

De outro modo, um processo de atualização de firmware é considerado seguro se fizer uso de mecanismos que garantam que somente firmware autênticos possam ser instalados no dispositivo [Nikolov 2018, Landwehr 2001, Russel and Gangemi 1991]. A autenticidade de um firmware pode ser verificada, por exemplo, por meio da criptografia de chave pública. Nesse caso, a chave pública do emissor do firmware deve ser inserida na memória do dispositivo e se deve garantir que a mesma não possa ser substituída por outra chave sem a aprovação do fabricante do dispositivo. Essa garantia pode ser atingida utilizando um hardware seguro, como um Secure Element (SE) ou um Trusted Execution Environment (TEE), que proveem armazenamento e execução segura de aplicações e dados, prevenindo que ataques que visam obter ou modificar uma informação sejam bem-sucedidos [Bouazzouni et al. 2018]. Por fim, no processo de atualização se deve encaminhar o novo firmware juntamente com uma assinatura que fora gerada pela chave privada, par da chave pública que está embarcada no dispositivo.

Fabricantes de microcontroladores disponibilizam soluções proprietárias para a atualização de firmware e que podem ser utilizadas por desenvolvedores. Essas soluções proveem um conjunto de APIs que devem ser utilizadas para que o desenvolvedor integre o bootloader do dispositivo às soluções de atualização de firmware. Entretanto, essas soluções não são interoperáveis entre os fabricantes, podendo ainda haver casos em que uma solução pode ser limitada a famílias específicas de microcontroladores dentro de um mesmo fabricante, de forma que o desenvolvedor seja obrigado a alterar integralmente o seu projeto de bootloader cada vez que utilizar um microcontrolador diferente [STMicroelectronics 2020b]. Por outro lado, uma solução de atualização de firmware genérica permite que fabricantes de dispositivos possam utilizar uma mesma implementação de bootloader ao mesmo tempo que microcontroladores diferentes sejam utilizados, sem que haja a necessidade de alterar o projeto do bootloader como um todo.

Este trabalho apresenta uma solução genérica para atualização de firmware de dispositivos embarcados de forma segura e confiável. A solução disponibiliza um projeto de bootloader que pode ser reaproveitado em diferentes microcontroladores, uma vez que o mesmo foi organizado em um conjunto de classes abstratas, sendo necessário apenas especializar os métodos que fazem a interação com o hardware dos microcontroladores, por exemplo, métodos que fazem leitura e escrita na memória de programa do microcontrolador. Uma implementação de referência foi realizada utilizando o microcontrolador 
STM32L562QE [STMicroelectronics 2020c], a qual permitiu demonstrar que a solução proposta é capaz de atualizar o firmware do dispositivo de forma que apenas firmware autênticos fossem instalados, ao passo que o dispositivo não entrou em um estado de execução não previsto, mesmo quando uma atualização não fora concluída com êxito.

Este trabalho está organizado da seguinte forma: na Seção 2 são apresentados trabalhos relacionados a este; a Seção 3 apresenta a proposta de atualização de firmware deste trabalho; na Seção 4 é apresentada a implementação realizada, enquanto que os experimentos e resultados obtidos são apresentados na Seção 5; por fim, na Seção 6 são apresentadas as conclusões obtidas.

\section{Trabalhos relacionados}

Em [Jain et al. 2016] é proposta uma solução de atualização de firmware que faz uso de criptografia simétrica para cifrar o firmware a ser transmitido e assim garantir a propriedade de confidencialidade. O firmware é transmitido para o dispositivo em blocos e é feito uso do Cyclic Redundancy Check (CRC) para para verificar a integridade de cada bloco. Os autores optaram por segmentar a memória do microcontrolador em duas partições, uma para conter o firmware em execução e outra temporária, para armazenar o novo firmware a ser recebido. Essa abordagem permite que o dispositivo sempre consiga iniciar um firmware, mesmo que uma tentativa de atualização tenha sido encerrada com falha.

O trabalho de [Nikolov 2018] tem como principal foco a proposição de uma solução para transmissão do novo firmware por meio da comunicação sem fio, ou Firmware-over-the-air (FOTA). Neste cenário, o dispositivo possui conectividade com a Internet e assim é capaz de iniciar o processo de atualização de firmware de forma automática. Para garantir a confiabilidade do processo de atualização, o autor optou pela abordagem de segmentação da memória do microcontrolador em duas partições e o uso de soma de verificação (checksum) para verificar a integridade do firmware recebido. Porém, o trabalho não faz uso de mecanismos para garantir a autenticidade deste firmware.

Em [Dhobi et al. 2019] é feito uso de TEE como hardware seguro e as rotinas do processo de atualização de firmware foram dividas em duas aplicações, uma armazenada no ambiente seguro e outra no ambiente não seguro. No ambiente seguro estão as rotinas para verificar a autenticidade e integridade do firmware e para isso é feito uso de criptografia de chave pública RSA combinada com o algoritmo de resumo criptográfico SHA-256. Na área não segura estão as rotinas para recebimento do firmware via FOTA, uma vez que o ambiente seguro do TEE não possui conectividade com a Internet.

No presente trabalho optou-se pelo uso da criptografia de chave pública para garantir a integridade e autenticidade do novo firmware, contudo a cifragem do mesmo não foi considerada como um requisito essencial. Assim, foi proposto o uso de hardware seguro para o armazenamento da chave pública do emissor do firmware, bem como de todo o código bootloader. Com relação a confiabilidade do processo de atualização, ou seja, a garantia de que o dispositivo não ficará inutilizado após uma tentativa falha de atualização, este trabalho propõe uma máquina de estados e uma organização de classes para implementação do bootloader permitindo a ao desenvolvedor escolher entre a abordagem com uma única partição para armazenamento do firmware e a abordagem com duas partições, sendo uma partição temporária para armazenamento do novo firmware . 
Dessa forma, acredita-se que a presente proposta seja adequada até mesmo para dispositivos com pouca área de armazenamento, mas ainda assim garantindo uma atualização segura e confiável.

\section{Solução para atualização de firmware de forma segura e confiável}

A solução de atualização de firmware apresentada neste trabalho é destinada a dispositivos com sistemas embarcados compostos por componentes com recursos de armazenamento e processamento limitados (e.g. microcontroladores) e com função específica. A proposta consiste na definição de: i) um conjunto mínimo de componentes de hardware, bem como sua organização; ii) os mecanismos e artefatos essenciais para garantir as propriedades de integridade e autenticidade do firmware, além da confiabilidade do processo de atualização; iii) a estruturação do arquivo binário que conterá o novo firmware a ser enviado ao dispositivo; iv) organização e conjunto de funcionalidades do bootloader.

\subsection{Componentes de hardware}

O hardware do dispositivo deve garantir as seguintes prerrogativas para o perfeito funcionamento do bootloader e da proposta aqui apresentada: i) armazenamento seguro garantir que as informações essenciais utilizadas pelo bootloader durante os processos de atualização e inicialização do dispositivo sejam acessadas com exclusividade pelo bootloader, que não estejam expostas para o firmware e que não possam ser extraídas de maneira não autorizada; ii) garantia da imutabilidade do bootloader - o mesmo não deve ser alterado após ser inserido no dispositivo; iii) a execução do bootloader deve ser prioritária - este sempre deve ser o primeiro código a entrar em execução no dispositivo a cada inicialização.

A garantia de exclusividade de leitura e escrita pode ser alcançada com a utilização de hardware seguros. É possível integrar um Secure Element (SE) em conjunto com um microcontrolador não seguro, de modo que um bootloader armazenado no microcontrolador busque as informações essenciais no elemento seguro sempre que necessário. Uma segunda organização possível consiste em usar um Trusted Execution Environment (TEE), sendo possível guardar no ambiente de armazenamento seguro, além do material criptográfico sensível, o próprio bootloader. Neste cenário, tanto o bootloader quanto as informações essenciais aos processos de atualização e inicialização são armazenadas e executadas de forma isolada ao firmware do dispositivo. Na Figura 1(a) é apresentada a organização de hardware do dispositivo utilizando um microcontrolador não seguro em conjunto com um SE, enquanto que na Figura 1(b) é apresentada uma organização de hardware do dispositivo utilizando um TEE, sendo esta última a organização utilizada na implementação realizada e discutida na Seção 4.

O firmware do dispositivo deve ser armazenado em uma região de memória na qual o bootloader possa ler e escrever. Essa região, também chamada de partição, deve ser definida de acordo com a área de armazenamento que o dispositivo possui. Em dispositivos com pouco espaço de armazenamento, deve-se definir uma única partição e a cada tentativa de atualização o bootloader deverá sobrescrever o conteúdo dessa partição com o novo firmware, aqui chamado de firmware candidato, e se o processo ocorrer com sucesso, então o firmware candidato deverá ser executado na próxima inicialização do dispositivo. Contudo, caso a atualização não seja bem-sucedida, então o bootloader não 
deve colocar o firmware candidato em execução e deverá indicar ao usuário (e.g. led piscando em vermelho) que alguma ação precisa ser tomada.

Quando a área de armazenamento for grande o suficiente para permitir a criação de duas partições, então pode ser definido uma partição de execução, a qual conterá o firmware a ser iniciado quando dispositivo for energizado, e outra partição na qual o firmware candidato é armazenado durante o processo de atualização. Dessa forma, se o processo de atualização ocorrer com sucesso, então o bootloader faz o chaveamento de partições, indicando que a partição de atualização será agora a partição de execução. Essa abordagem tem como vantagem, em relação a abordagem com partição única, a possibilidade de que o dispositivo nunca ficará em um estado de exceção que demande uma ação do usuário. Ou seja, é possível iniciar o firmware antigo, mesmo que o processo de atualização tenha sido finalizado sem sucesso.

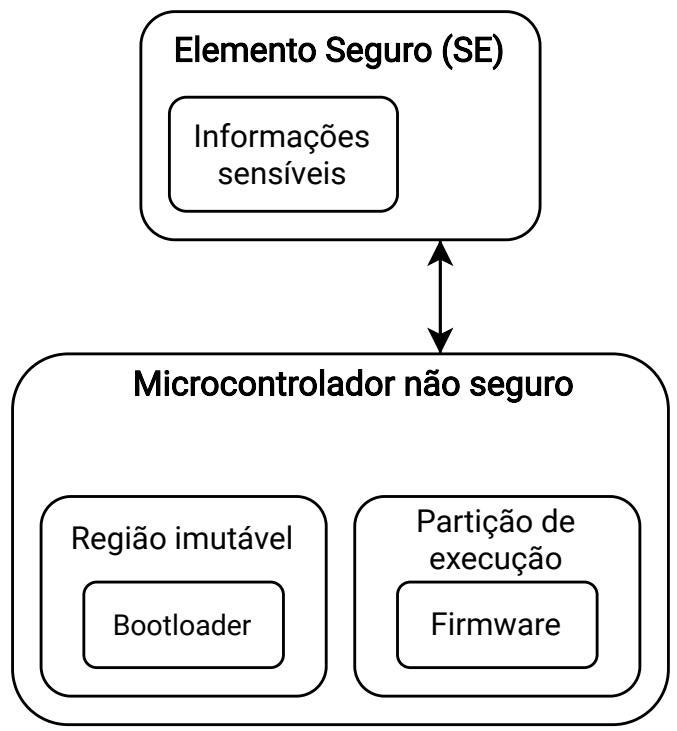

(a) Organização com SE.

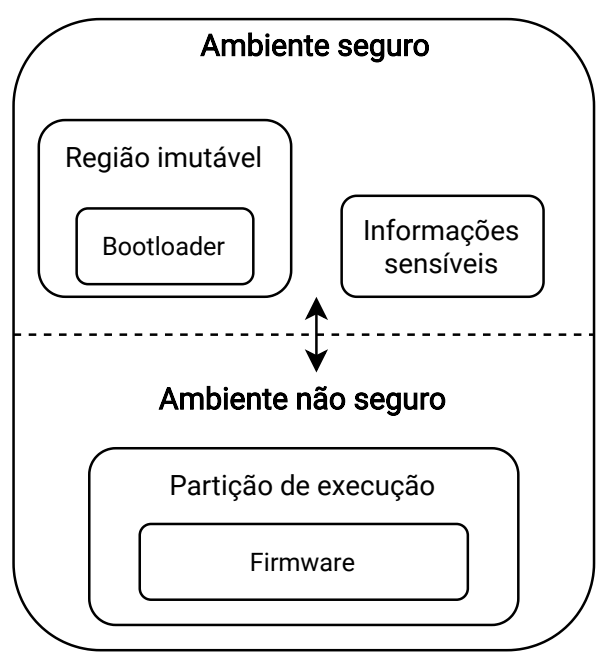

(b) Organização com TEE.

Figura 1. Organizações de hardware do dispositivo.

\subsection{Mecanismos para garantir e integridade e autenticidade do firmware}

O bootloader precisa garantir que o firmware instalado na partição de execução esteja íntegro antes que possa executá-lo, e na presente proposta isso é alcançado utilizando resumos criptográficos (hash). Ao receber um firmware candidato e garantir que o mesmo está íntegro, armazena-se o hash desse firmware no ambiente seguro. A cada processo de inicialização o bootloader deve calcular o hash do firmware presente na partição de execução e verificar a correspondência deste com o hash mantido no ambiente seguro.

A autenticidade do firmware, ou seja, a determinação que o mesmo foi gerado por uma parte autorizada (e.g. fabricante do dispositivo) é obtida na presente proposta por meio da criptografia de chave pública. Neste caso, a chave pública do fabricante deve ser inserida, em tempo de manufatura, no ambiente seguro do dispositivo. No processo de atualização, juntamente com o firmware candidato, deve-se encaminhar a assinatura digital gerada sobre o mesmo com a chave privada, par daquela mantida no dispositivo. A estrutura do binário a ser enviado no processo de atualização é apresentada na Subseção 3.3. 
Uma vez que foi verificada a integridade e autenticidade do firmware candidato e o mesmo foi gravado na partição de execução, o processo de inicialização não precisará mais verificar a autenticidade do firmware a cada vez que o dispositivo for energizado.

Em alguns cenários, pode ser desejada a garantia de que somente novas versões de um firmware possam ser instaladas no dispositivo. Ou seja, não é desejado que o usuário possa instalar uma versão antiga, mesmo que essa tenha sido emitida e esteja assinada com a chave privada do fabricante do dispositivo. Nesta proposta está previsto o envio de um valor, juntamente com o binário do firmware, para representar a versão do firmware. As versões de cada firmware candidato devem ser crescentes e esta informação deve ser utilizada pelo bootloader no momento da atualização do dispositivo, impedindo que o firmware candidato seja instalado caso sua versão seja inferior ao firmware já armazenado no dispositivo. A versão do firmware candidato deve ser assinada pelo fabricante do dispositivo em conjunto com o binário do firmware. Dessa forma, o bootloader poderá ter certeza que a assinatura que vai ser verificada tem relação exclusivamente com o firmware candidato e o valor da versão que foram recebidos.

A presente proposta não restringe quais algoritmos de resumo criptográfico e de chave pública devem ser usados, ficando a critério do fabricante do dispositivo e de acordo com as capacidades de cada microcontrolador, tendo em vista que alguns microcontroladores possuem aceleradores criptográficos para algoritmos específicos.

\subsection{Estrutura do arquivo binário com o firmware candidato}

O bootloader precisa receber, além do firmware candidato, a versão deste firmware e a assinatura digital realizada sobre ambos. Ou seja, tem-se aqui três informações distintas e que poderiam ser enviadas em três transmissões sequenciais, e assim a necessidade da definição de um protocolo o qual indicaria a ordem das informações que seriam transmitidas e o que deveria ser feito caso não fosse recebida a informação esperada. Nesta proposta, optou-se pela simplicidade do processo de transmissão, e assim foi criado uma estrutura para que em um único binário estivessem a assinatura digital, a versão do firmware e o próprio firmware candidato. Ao bootloader cabe fazer a separação desse binário para conseguir obter cada informação.

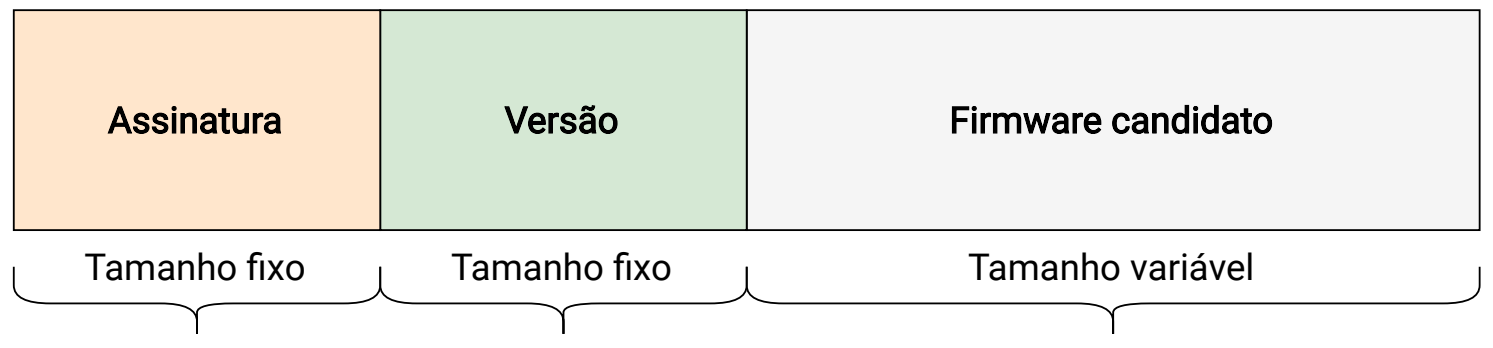

Figura 2. Estrutura do binário proposto.

Na Figura 2 é apresentada a estrutura de binário proposta neste trabalho. A versão do firmware e a assinatura digital possuem tamanhos fixos, sendo a última em função do algoritmo de assinatura digital escolhido. $\mathrm{O}$ tamanho fixo dessas informações permite ao bootloader separar facilmente o binário recebido e obter as informações necessárias para os processos de atualização e inicialização, enquanto que os últimos bytes do binário são referentes ao firmware candidato. 


\subsection{Organização e funcionalidades do bootloader}

Na Figura 3 é apresentada uma das contribuições deste trabalho, um diagrama de classes conceitual UML [OMG 2017] para guiar fabricantes de dispositivos na implementação de seus bootloader. Ciente que a implementação do bootloader é dependente das ferramentas e da arquitetura de cada microcontrolador, todas as classes propostas são abstratas e assim devem ser especializadas e implementadas pelo fabricante do dispositivo. Um diagrama de classes UML na perspectiva de implementação, bem como a codificação dessas classes abstratas na linguagem $\mathrm{C}++$ estão disponíveis no GitHub ${ }^{1}$.

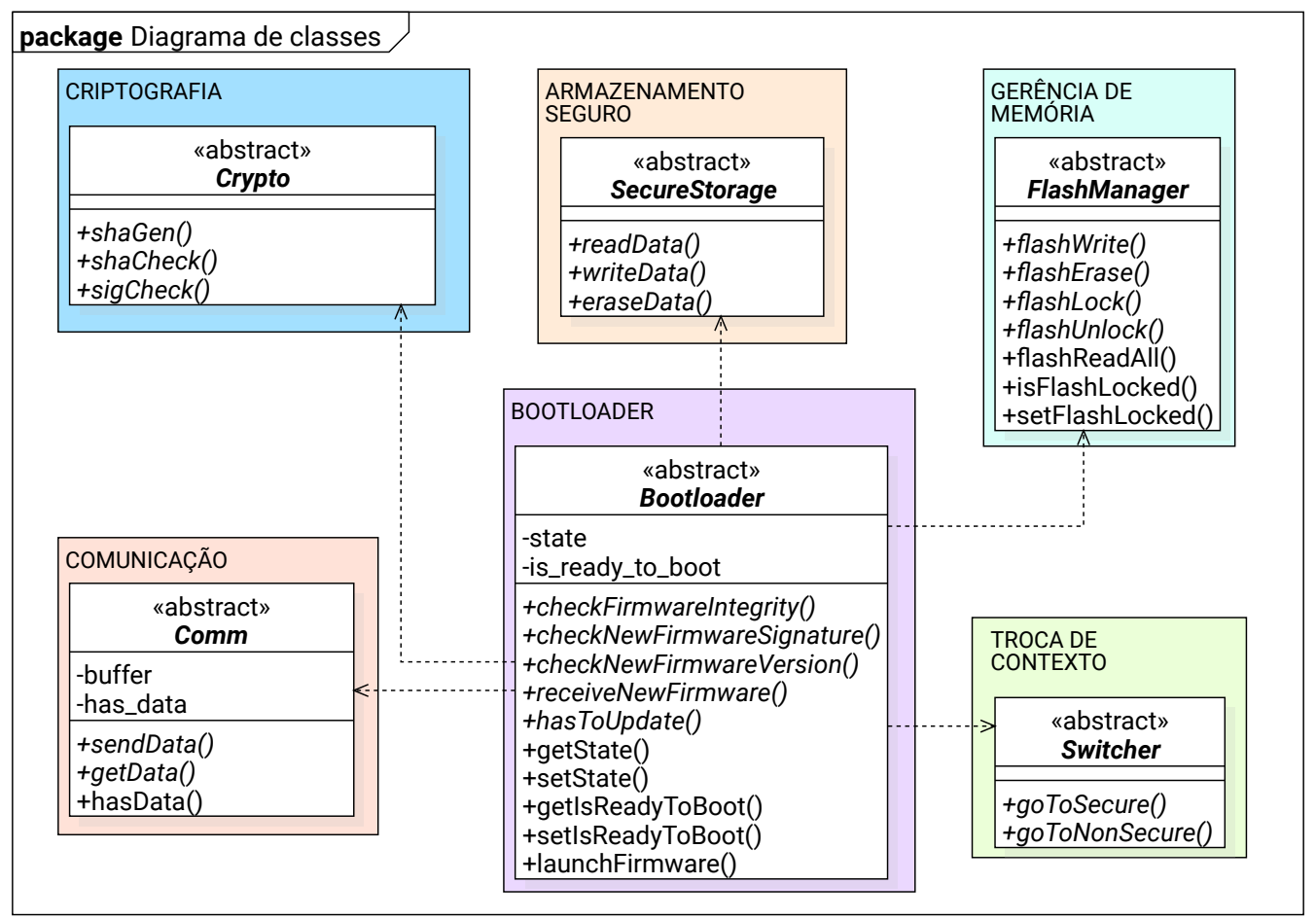

Figura 3. Diagrama de classes UML do bootloader proposto.

A classe Bootloader é responsável por implementar todo o comportamento do bootloader e, para melhor organização, depende das demais classes para executar as operações de criptografia (gerar e verificar assinaturas e resumos), armazenamento seguro (ler e escrever no ambiente seguro), gestão de memória (ler e escrever nas partições), comunicação (enviar e receber dados de acordo com a tecnologia usada, e.g. USB) e troca de contexto.

A classe para troca de contexto (Switcher) tem por objetivo permitir encerrar a execução do bootloader e colocar o firmware do dispositivo em execução, podendo também ser utilizada para a operação inversa. Assim, o firmware do dispositivo também deve apresentar uma implementação para esta classe. Apenas dois métodos são propostos nesta classe: um para a troca de contexto entre o bootloader e o firmware e outro para que o firmware avise ao bootloader que o processo de atualização de firmware deve ser iniciado.

\footnotetext{
${ }^{1}$ https://github.com/ifsc-saojose/secure-firmware-update/tree/master/artigo-wticg/ classes-abstratas
} 
O bootloader proposto foi modelado a partir de uma máquina de estados finita (i.e. Finite State Machine - FSM), apresentada na Figura 4, na qual três estados são definidos: verificação, confirmação e atualização. O estado atualização, por sua vez, é dividido em quatro subestados: preparação, recepção, validação e instalação.

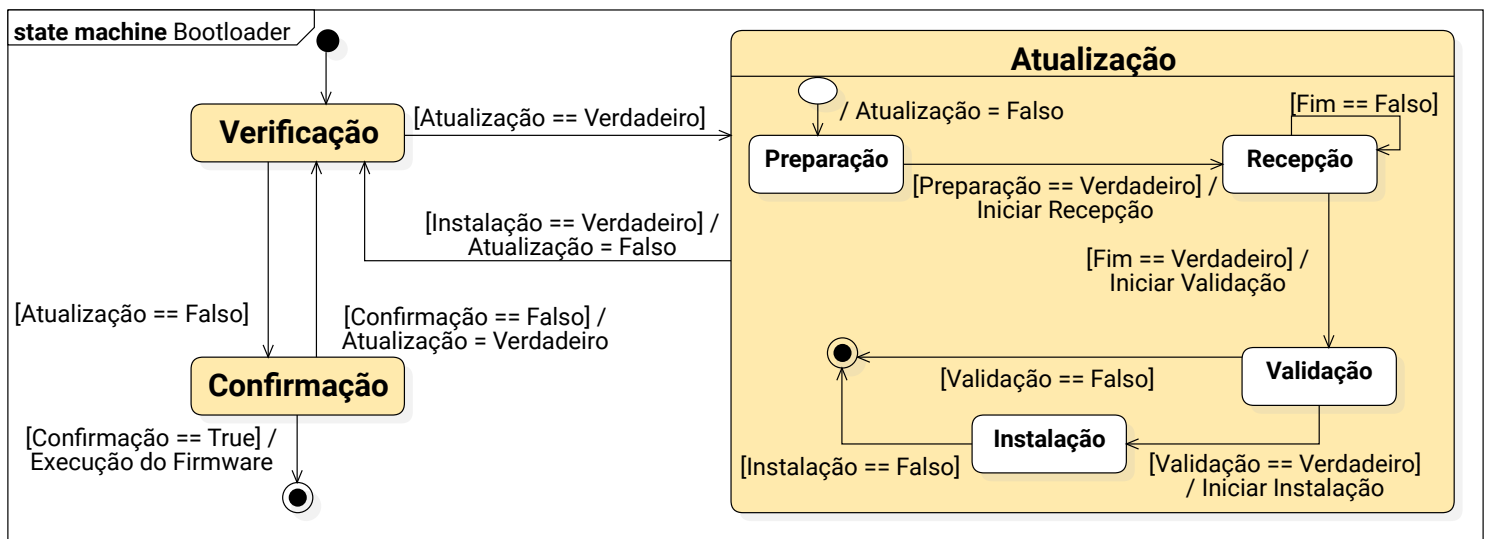

Figura 4. Máquina de estados finitos UML para o bootloader.

No estado verificação o bootloader verifica se deve iniciar o processo de atualização de firmware. Assim, consulta a variável atualização que está armazenada no ambiente seguro. Se estiver como verdadeiro, então irá para o estado atualização. Se estiver como falso, então irá para o estado confirmação onde é feita a verificação de integridade do firmware presente na partição de execução. Se estiver íntegro, é feito o chaveamento de contexto, colocando o firmware em execução. Se o firmware não estiver íntegro, então o bootloader deve atribuir o valor verdadeiro na variável atualização, indicando assim a necessidade de atualização, e retornar ao estado inicial (verificação).

O firmware em execução deve possuir uma funcionalidade (a ser invocada pelo usuário) que permita atribuir o valor verdadeiro na variável atuali zação para indicar ao bootloader que este deverá ir para o estado de atualização assim que for feito o chaveamento de contexto, ou seja, quando o firmware passar a execução para o bootloader. Somente o bootloader será capaz de alterar para falso o conteúdo da variável atualização e este sempre o fará a partir do momento que entrar no estado atualização.

No subestado preparação, o bootloader atribui o valor falso na variável atualização e prepara o dispositivo para o recebimento do binário contendo o firmware candidato. A principal ação nesse estado é a limpeza da região de memória que irá armazenar o firmware candidato contido no binário a ser recebido, bem como a limpeza das regiões de memória no ambiente de armazenamento seguro que irão armazenar a assinatura e versão do firmware candidato, que também estão contidos no binário. Uma vez que a limpeza foi finalizada, então o bootloader passa para o estado recepção onde o binário é recebido e armazenado na participação de atualização.

Assim que encerrar o recebimento e armazenamento do binário, o bootloader passa para o estado validação, onde acontecem as operações para verificar a integridade e autenticidade do firmware recebido, bem como se o número da versão recebido é superior ao atual número armazenado no ambiente seguro. Caso qualquer uma dessas verificações resulte em insucesso, então o bootloader aborta o processo de atualização e deve indicar ao usuário que a atualização não foi bem-sucedida, além de permanecer neste estado 
até que o usuário reinicie o dispositivo. Por outro lado, se as validações resultarem em sucesso, então o bootloader passa para o estado instalação.

No estado instalação, o bootloader define que o firmware recém recebido é a partir de agora o firmware que deve ser executado no dispositivo, realizando o chaveamento entre as partições do dispositivo. Por fim, é gerado o resumo criptográfico sobre o binário do firmware recebido e persistido no ambiente seguro. Caso algum dos processos deste estado resulte em falha, o bootloader deve abortar o processo de atualização e indicar ao usuário que a atualização não foi bem-sucedida, devendo ficar neste estado até que o usuário reinicie o dispositivo. Por fim, caso o processo de geração do resumo criptográfico e a instalação do firmware tenha sido bem-sucedida, o bootloader deve retornar ao estado verificação.

\section{Implementação da solução proposta}

A fim de verificar se a organização de classes e o comportamento do bootloader propostos são factíveis, foi feita uma implementação utilizando o microcontrolador STM32L562QE, da fabricante STMicroelectronics, com o kit de desenvolvimento STM32L562-DK Discovery.

Este microcontrolador é composto pelo o TrustZone, a implementação de um TEE da empresa $A R M$, o qual foi utilizado como ambiente de armazenamento seguro para as informações essenciais aos processos de atualização e inicialização do dispositivo. Com o TrustZone é possível fazer uma divisão da memória de programa do microcontrolador em área segura e área não segura. Um código sendo executado na área não segura é totalmente isolado e não possui acesso à área segura, a não ser pela possibilidade de chamadas feitas para funções armazenadas em uma região chamada Non-Secure Callable (NSC). Essa região serve para armazenar código com funções de interface, as quais permitem a transição controlada de um ambiente não seguro para um ambiente seguro. Essas chamadas precisam ser pré-definidas e acordadas em tempo de compilação. Por outro lado, um código executado na área segura tem acesso a toda memória de programa do microcontrolador [STMicroelectronics 2020c].

O TrustZone provê um mecanismo de segurança chamado Write Protection (WRP), que permite que uma área específica da memória de programa seja imutável. Neste caso foi feito uso do WRP nas regiões que armazenam o bootloader e a chave pública do emissor do firmware. Com o TrustZone também foi possível definir um ponto único de início de execução, sendo este o endereço de memória o qual o bootloader foi armazenado.

\subsection{Implementação do bootloader}

Nesta seção é apresentado como cada classe abstrata presente na Figura 3 foi implementada. O microcontrolador usado conta com uma interface USB e essa foi usada como interface de comunicação para recebimento do binário contendo o firmware candidato. Foi implementada a classe USB Communications Device Class (CDC), subclasse Abstract Control Model (ACM) [USB-IF 2010], pois esta permite estabelecer uma comunicação serial entre os pares comunicantes de forma simples e o fabricante do microcontrolador disponibiliza uma biblioteca de fácil compreensão e utilização. 
O microcontrolador escolhido possui acelerador criptográfico em hardware que implementa criptografia Rivest-Shamir-Adleman (RSA) e criptografia de curvas elípticas. Optou-se por usar chaves criptográficas RSA com tamanho de 2.048 bits e o SHA-256 [NIST 2015] como algoritmo para resumos criptográficos (hash), uma vez que as bibliotecas criptográficas do fabricante forneciam suporte a estes.

O fabricante do microcontrolador oferece biblioteca para manipulação da memória de programa do mesmo. Esta biblioteca foi utilizada para a especialização da classe de gerência de memória desta proposta e aqui foi escolhida a abordagem com duas partições: partição de execução - que contém o firmware que deverá ser executado após o término da execução do bootloader; partição temporária - para armazenar o firmware candidato durante o processo de atualização.

A utilização do TEE como ambiente de armazenamento seguro permitiu utilizar a classe de gerência de memória como especialização da classe de armazenamento seguro (veja Figura 3). Neste caso, o acesso à área de memória segura pelo bootloader ocorre da mesma maneira que o acesso à área não segura, não sendo necessário prover uma implementação específica para trabalhar com a memória segura. Se fosse feito uso de uma organização com elemento seguro (SE) e não TEE, então seria necessário prover uma implementação específica da classe de armazenamento seguro que interagisse com o hardware externo.

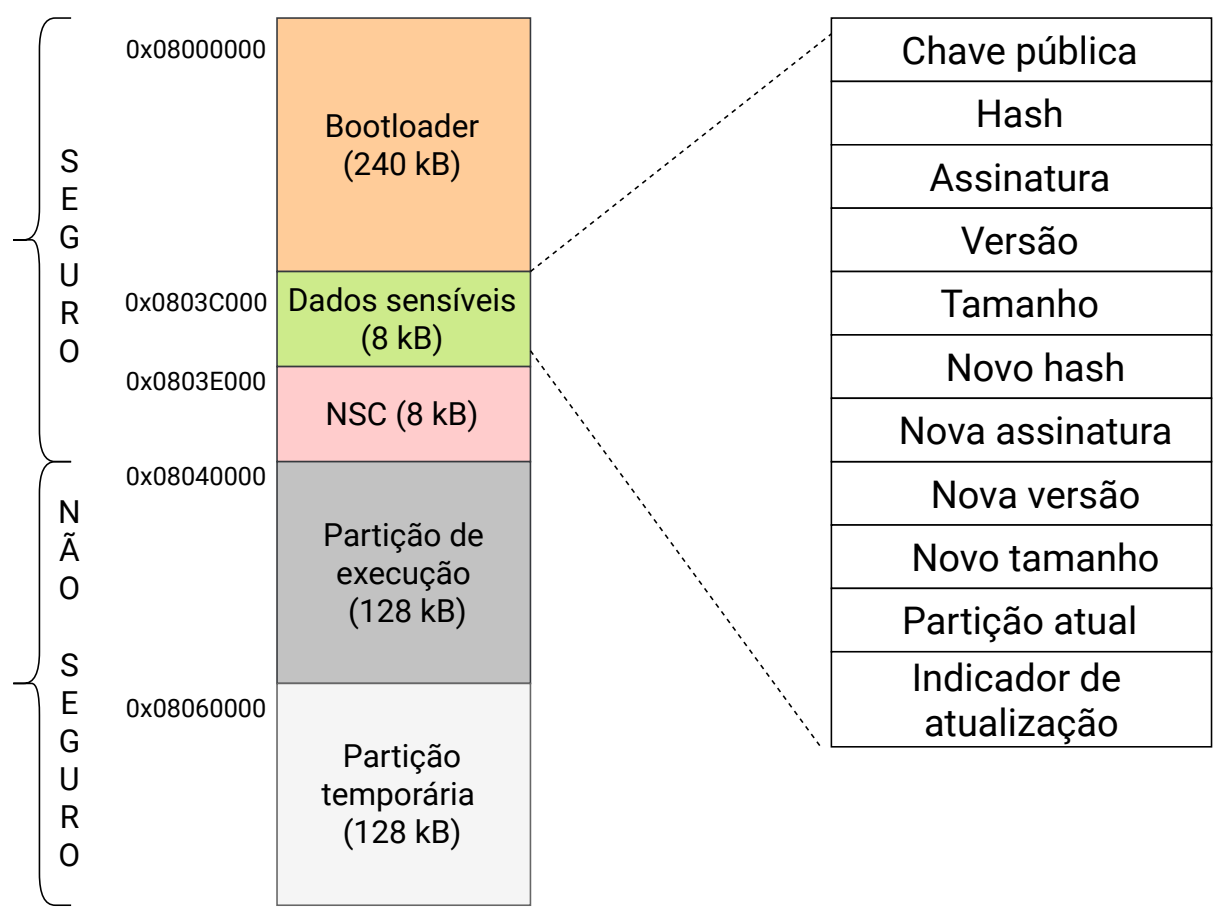

Figura 5. Segmentação da memória de programa do microcontrolador.

Na Figura 5 é apresentada a segmentação da memória de programa do microcontrolador para armazenar o código do bootloader, dados sensíveis, as rotinas Non-Secure Callable (NSC), bem como as áreas destinadas para as partições de execução e temporária. Na figura é possível observar em qual ambiente de execução, seguro e não seguro, cada informação é armazenada. 
A implementação da classe switcher consistiu em uma função que faz o salto da memória de programa segura (bootloader) para a memória de programa não segura, colocando o firmware em execução como recomendado pelo fabricante do microcontrolador [STMicroelectronics 2020a].

Por fim, a classe bootloader foi especializada a partir das classes já discutidas, de modo que alguns métodos desta classe são dependentes das demais especializações, por exemplo, o método que verifica a integridade do firmware atual, que depende da classe de criptografia.

\subsection{Implementação do firmware}

A fim de garantir que o firmware possa avisar ao bootloader sobre uma tentativa de atualização, a implementação do firmware especializou a classe abstrata de troca de contexto (veja Figura 3). A especialização desta classe se deu a partir de uma chamada NSC, fazendo assim uso da Application Programming Interface (API) compartilhada entre as áreas segura (bootloader) e não segura (firmware. A implementação da chamada NSC foi feita na área segura e na área não segura foi feita a invocação dessa API compartilhada.

\section{Experimentos e resultados}

Foram realizados quatro experimentos para verificar se o modelo e organização propostos, bem como a implementação no microcontrolador STM32L562QE, gerariam os resultados esperados. Para verificar se a atualização de firmware acontecia com sucesso, foram desenvolvidas duas versões de firmware, versão 01 e versão 02. Cada firmware tinha um comportamento distinto de forma que fosse fácil identificar qual firmware estava em execução após uma tentativa de atualização. A versão 01 faz com que o led do kit de desenvolvimento pisque a cada 100 milissegundos, enquanto que a versão 02 faz com que o led pisque a cada 1.000 milissegundos. Os experimentos conduzidos foram:

1. Transmissão completa e íntegra de todos os dados necessários para que a atualização ocorresse com sucesso;

2. Tentativa de atualizar um firmware com versão inferior à versão instalada no dispositivo;

3. Tentativa de atualizar com uma assinatura digital inválida sobre o binário;

4. Tentativa de atualizar com a transmissão sendo interrompida antes da transferência completa do binário.

Para o experimentos 1, 2 e 3 foi feito o seguinte procedimento: com o dispositivo energizado e com o firmware em execução, pressionou-se o botão do kit de desenvolvimento para iniciar o processo de atualização. Uma vez que o bootloader entrou em execução, então foi iniciada a transmissão do binário (veja Figura 2) pela interface USB do kit de desenvolvimento e aguardou-se que essa fosse executada por completo. Para o experimento 4, o dispositivo era reiniciado antes que a transferência completa do binário pudesse ser concluída com sucesso. Na Tabela 1 são apresentados os resultados esperados e obtidos com cada experimento.

No experimento 1, o dispositivo estava com o firmware versão 01 instalado e após o recebimento do binário o bootloader do dispositivo validou a versão e assinatura e instalou o firmware com sucesso. Por fim, o bootloader encerrou sua execução, validando a integridade do firmware e colocando o firmware versão 02 em execução, finalizando o experimento com sucesso. 
Tabela 1. Resumo dos experimentos realizados

\begin{tabular}{rlllll}
\hline Experimento & Assinatura & Versão & Transmissão & \multicolumn{2}{c}{ Resultado } \\
& & & & Esperado & Obtido \\
\hline 1 & válida & superior & completa & sucesso & sucesso \\
2 & válida & inferior & completa & insucesso & insucesso \\
3 & inválida & superior & completo & insucesso & insucesso \\
4 & válida & superior & incompleta & insucesso & insucesso \\
\hline
\end{tabular}

No experimento 2, o dispositivo estava com o firmware versão 02 instalado e recebeu o binário com o firmware versão 01. Ao final da transmissão, o bootloader do dispositivo verificou que a versão recebida era inferior à versão do firmware já instalado e assim interrompeu a tentativa de atualização. O bootloader indicou ao usuário que o processo de atualização não foi bem-sucedido e, após a reinicialização do dispositivo, o firmware versão 02 voltou a ser executado, conforme o esperado.

Para o experimento 3, o dispositivo estava com o firmware versão 01 instalado e recebeu o binário com o firmware versão 02 , porém contendo uma assinatura que não fora gerada com a versão e firmware candidato contidos no binário. O bootloader, ao constatar que a assinatura era inválida, interrompeu a tentativa de atualização, indicou ao usuário que o processo de atualização não foi bem-sucedido e, após a reinicialização do dispositivo, o firmware versão 01 voltou a ser executado, conforme o esperado.

Por fim, no experimento 4, com o firmware versão 01 sendo executado no dispositivo, o processo de atualização foi iniciado, sendo transmitido ao dispositivo binário com o firmware versão 02 e com assinatura gerada pela chave par da chave pública contida no dispositivo. Antes que o binário fosse recebido por completo pelo dispositivo, foi retirada a alimentação do dispositivo e quando este voltou a ser energizado, o bootloader colocou o firmware versão 01 em execução e ignorou os dados corrompidos na partição temporária, como o esperado.

\section{Conclusões}

Este trabalho apresentou uma proposta de atualização de firmware voltada para dispositivos utilizados em sistemas embarcados, de modo que essa atualização permita que apenas firmware autênticos possam ser instalados, além de garantir que o dispositivo não fique inutilizável após o processo de atualização caso a atualização não seja finalizada com sucesso. Também foi proposta uma organização de classes para guiar a implementação do bootloader em microcontroladores com diferentes arquiteturas, sendo necessário apenas especializar as classes que interagem com o hardware de cada microcontrolador.

Foi feita uma implementação da organização proposta no microcontrolador STM32L562QE que possui um TEE, sendo este o ambiente seguro no qual o bootloader e as informações sensíveis aos processos de atualização e inicialização do dispositivo foram armazenados. Na implementação, optou-se pela abordagem com duas partições (execução e temporária) para permitir que o dispositivo possa se recuperar caso alguma tentativa de atualização do firmware do dispositivo termine sem sucesso.

Quatro experimentos foram conduzidos a fim de validar o modelo proposto, bem 
como validar a implementação específica para o microcontrolador STM32L562QE. Os experimentos permitiram demonstrar que a solução de atualização de firmware e bootloader propostos, bem como a respectiva implementação, permitem que a atualização do firmware do dispositivo ocorra somente quando um firmware autêntico é transferido ao mesmo, além de não inutilizá-lo quando uma tentativa de atualização não é finalizada com sucesso.

Como proposta de trabalhos futuros, sugere-se implementar a solução apresentada neste trabalho utilizando um microcontrolador não-seguro acoplado com um elemento seguro, de modo a validar a solução de atualização de firmware para esta organização de hardware. Sugere-se também implementar a solução utilizando apenas um partição de armazenamento de firmware.

\section{Referências}

[Beningo 2015] Beningo, J. (2015). Bootloader design for microcontrollers in embedded systems.

[Bouazzouni et al. 2018] Bouazzouni, M. A., Conchon, E., and Peyrard, F. (2018). Trusted mobile computing: An overview of existing solutions. Future Generation Computer Systems, 80:596 - 612.

[Dhobi et al. 2019] Dhobi, R., Gajjar, S., Parmar, D., and Vaghela, T. (2019). Secure firmware update over the air using trustzone. In 2019 Innovations in Power and Advanced Computing Technologies (i-PACT), volume 1, pages 1-4.

[Jain et al. 2016] Jain, N., Mali, S. G., and Kulkarni, S. (2016). Infield firmware update: Challenges and solutions. In 2016 International Conference on Communication and Signal Processing (ICCSP), pages 1232-1236.

[Landwehr 2001] Landwehr, C. (2001). Computer security. International Journal of Information Security, 1:3-13.

[Nikolov 2018] Nikolov, N. (2018). Research firmware update over the air from the cloud. In 2018 IEEE XXVII International Scientific Conference Electronics - ET, pages 1-4.

[NIST 2015] NIST (2015). Secure Hash Standards. National Institute of Standards and Technology.

[OMG 2017] OMG (2017). Omg unified modeling language (omg uml).

[Russel and Gangemi 1991] Russel, D. and Gangemi, G. T. (1991). Computer Security Basics. O'Reilly \& Associates.

[STMicroelectronics 2020a] STMicroelectronics (2020a). Getting started with projects base on the stm3215 series in stm32cubeide.

[STMicroelectronics 2020b] STMicroelectronics (2020b). Overview of secure boot and secure firmware update solution on arm trustzone stm3215 series microcontrollers.

[STMicroelectronics 2020c] STMicroelectronics (2020c). Reference manual - stm321552xx and stm321562xx advanced arm-based 32-bit mcus.

[USB-IF 2010] USB-IF (2010). Universal Serial Bus Class Definitions for Communications Devices. USB-IF. 